\title{
Progress in the Development of Radio-Cherenkov Neutrino Detectors
}

\author{
Steven W. Barwick ${ }^{1}$ \\ University of California \\ Irvine, CA 92617, USA \\ E-mail: sbarwick@uci.edu
}

Evidence is growing for the existence of a diffuse flux of astrophysical neutrinos with energies up to a few $\times 10^{15} \mathrm{eV}$. This has spurred considerable interest in developing new techniques that can extend the search to even higher neutrino energies. Promising new efforts over the past halfdecade focus on the radio-Cherenkov technique in polar regions with cold, highly transparent ice. I will describe the broad, complementary physics goals of these telescopes with design sensitivities as least a factor 10 better than current limits for energies $>10^{17.5} \mathrm{eV}$. One important goal focuses on a measurement of the cosmological neutrino flux, whose existence is relatively secure but also expected to be quite small even under the best of circumstances. Fortunately, the inherent cost efficiencies of radio-Cherenkov techniques suggest far greater boosts in sensitivity are possible. After briefly reviewing the progress by ANITA, ARA, and GNO collaborations, I discuss the first results from the ARIANNA Hexagonal Radio Array, completed in December 2014 and located on surface of the Ross Ice Shelf at a site about $110 \mathrm{~km}$ from McMurdo Station, Antarctica.

The 34th International Cosmic Ray Conference

30 July- 6 August, 2015

The Hague, The Netherlands

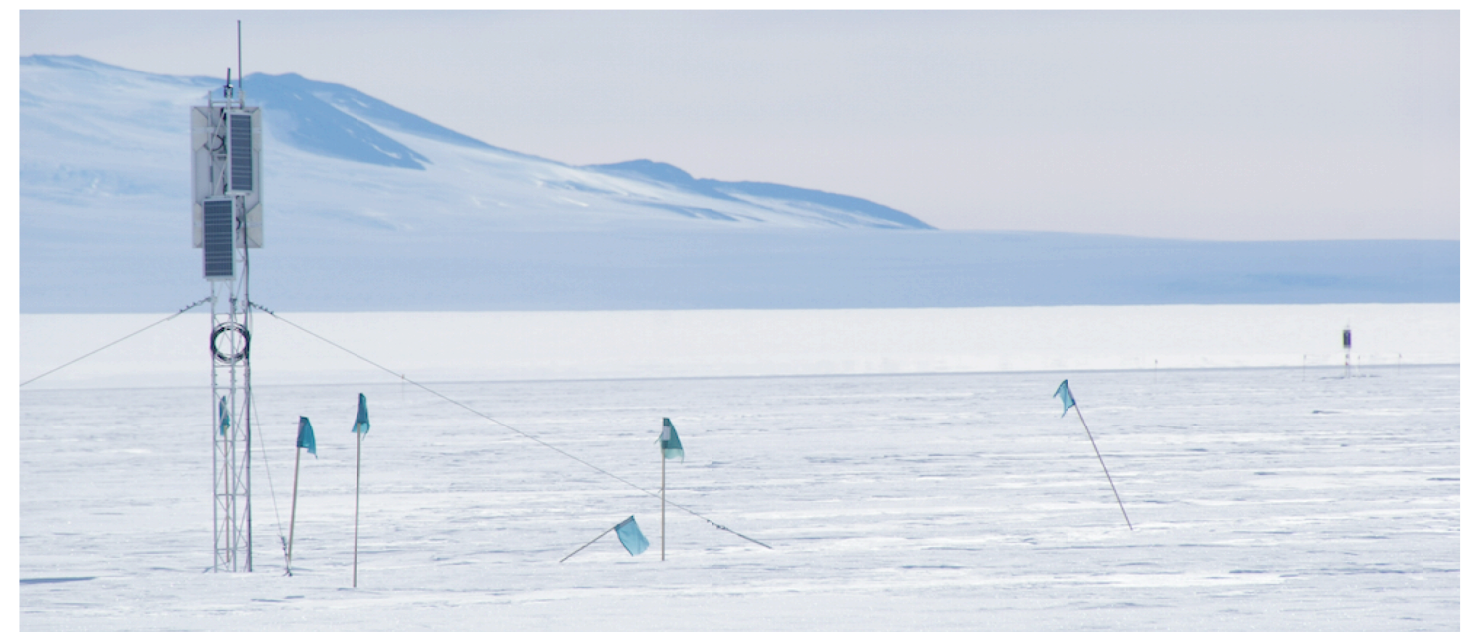

\section{${ }^{1}$ Speaker}




\section{Introduction}

One of the foremost questions in astronomy and astrophysics concerns the identification and evolution of the cosmic accelerators that produce the highest energy (UHE) cosmic rays [1]. What is the physics mechanism that is responsible for the production of the UHE cosmic rays and once accelerated, what additional interactions occur during propagation? The observation of UHE cosmogenic neutrinos that travel from their source undeflected by galactic and interstellar magnetic fields and unimpeded by interactions with the cosmic microwave background $(\mathrm{CMB})$ radiation may provide the critical insight to these questions. Even a nondetection may help constrain the elemental composition. However, uncertainties in the predicted cosmogenic neutrino fluxes have hindered past efforts to construct a detector with sufficient sensitivity to collect a statistically meaningful sample of events. Fortunately, due to their extreme energies, cosmogenic neutrinos are efficiently detected in dense, radio frequency (RF) transparent media via the Askaryan effect [2]. The vast expanses of cold ice in Antarctica, with its exceptional clarity to radio frequencies (RF), has been the host of several pioneering efforts to develop the Askaryan RF technique for neutrino astronomy.

During their travels from the most distant recesses of the Universe, the highest energy cosmic rays collide with photons in the microwave background to create "cosmogenic" or "GZK" neutrinos [3][4]; potent new messengers with energies between 10 17-20 eV. They provide a novel opportunity to help unravel the origins of the cosmic rays. Since neutrinos are uncharged and only interact weakly, they can travel unimpeded from the most distant sources and point back to their origin. In particular, GZK neutrinos are produced within a few tens of Mpc of the cosmic ray source, so it is expected that GZK neutrinos will point back with subdegree accuracy. In addition, the neutrino energy spectrum helps to break model degeneracy between source distribution and evolution [5], complementing the studies of cosmic ray detectors. More dramatically, the neutrino "beam" at extreme energies can be exploited to attack the question of what lies beyond our current understanding of the physical world, as defined by the standard model of elementary particle physics [1][6]. Neutrino telescopes can probe for physics beyond the standard model by measuring the neutrino cross-section at center of mass energies near $100 \mathrm{TeV}$ with good precision [7], or by discovering an unexpected feature in the energy spectrum [8][9]. Thus, the scientific promise of neutrino astronomy at extreme energies remains as exciting, compelling and elusive as ever.

Despite tremendous growth in instrumental sensitivity over the past decade, no detector has yet observed neutrinos with energies above $10 \mathrm{PeV}$ - due their small fluxes. To fully exploit this transformative cosmic messenger at extreme energies, defined as $\mathrm{E}>10 \mathrm{PeV}$, more capable detectors are required. The next generation of neutrino telescopes, specifically optimized for extreme energies, will dramatically improve the odds of success. During the past decade, Antarctica has emerged as one of the preferred locations to construct and operate high energy neutrino telescopes. Neutrinos interact so infrequently that a realistic detector must encompass or survey an enormous number of target nuclei, and the target medium must be transparent to the electromagnetic signals generated by the interaction. Several large projects (AMANDA [10], ANITA [11], IceCube [12], and RICE [13] and new concepts under development, such as ARIANNA [14], ARA [15], and GNO [16] ) exploit the fact that polar ice is transparent to 
radio and optical emission. Indirect evidence for cosmogenic neutrinos is provided by the Auger [17], HiRes [18] and Telescope Array [19][20] collaborations, who have reported flux suppression in cosmic ray spectra at the very highest energies. Suppression is commonly attributed to the GZK mechanism, but not without controversy (for example, see [21][22]).

When ultra-high energy neutrinos interact in a dense medium, such as the ice in the Ross Ice Shelf, the enormous cascade of secondary particles emit an intense sub-nanosecond pulse of coherent Cherenkov radiation at radio wavelengths. This emission mechanism, known as the Askaryan effect [2], was experimentally confirmed less than a decade ago in ice and other media to an accuracy within $20 \%$ [23][24]. Roughly speaking, the effect arises from the timevarying excess of negative charge that builds up as electrons from the ice medium are swept up by the development of the cascade [25]. The longer wavelengths of the broadband emission from the collective motion of the net charge will add coherently, producing a short duration, intense radio pulse. The shower dimensions determine the maximum frequency of coherent emission, typically about $5 \mathrm{GHz}$, but absorption in the media tend to limit the upper frequencies to $\sim 1 \mathrm{GHz}$. The balloon-borne ANITA payload [11] and the South Pole based RICE array [13] have exploited this effect to produce constraints on the extraterrestrial neutrino flux. Note that geomagnetic charge separation, which plays an important role in the radio emission from atmospheric cascades, can be neglected for cascades in dense media.

More recently, AMANDA-II [26], Auger [27] and HiRes [28] and IceCube [29][30] have searched for neutrinos in the extreme energy regime between $10^{16}$ and $10^{18} \mathrm{eV}$. The sensitivity of Auger and Telescope Array, which detect tau-neutrinos, and IceCube will improve with continued operation, but thus far cosmogenic neutrinos have not been observed after years of operation. This paper reviews several efforts to increase the sensitivity to neutrinos above $10^{17} \mathrm{eV}$ : ARIANNA [14], ARA [15], GNO [16] and GRAND [31], a new concept to observe radio emission from the atmospheric cascade of tau-decay. We will address the relative merits of the frontrunners in a later section.

\section{Science Objectives}

Greisen, Zatsepin, and Kuzmin (GZK) [3] first recognized that cosmic rays with energies in excess of $3 \times 10^{19} \mathrm{eV}$ readily interact with cosmic microwave photons and lose energy quickly, thereby limiting their propagation distance to the local supercluster. Despite several decades of progress in these calculations, there remains significant theoretical imprecision in the estimating the energy spectrum of cosmogenic neutrinos due to uncertainty associated with the elemental composition and injection spectra of the cosmic rays, source evolution, and cosmology.

This uncertainty is schematically illustrated in Fig. 1 by three gray patterned bands correspond to different classes of models: (1) all proton and proton-dominant "dip" models [32], (2) transition models that span a large range of assumptions associated with mixed composition, maximum acceleration energy and evolution of sources, and (3) the lowest band surveys iron dominant models. Current experimental efforts will only probe the most optimistic predictions dominated by protons. However, radio-Cherenkov neutrino telescopes will measure the energy spectrum and search for anisotropies. In addition, radio-based neutrino telescopes are uniquely sensitive to a mixed "light" Cosmic Ray composition with mean mass between Helium and 
Carbon at the highest energies, consistent with recent experimental data from Pierre Auger Observatory [33] and Telescope Array [34]. Even the absence of neutrinos would be quite important because the very lowest flux predictions arise from models that rely on interesting and nontraditional astrophysics. For example, the injected spectra must be unusually hard, the composition dominated by iron, and the maximum acceleration energy per nucleon must stop just short of the photonuclear fragmentation threshold [21]. From the astrophysics point of view, this may be the most exciting outcome.

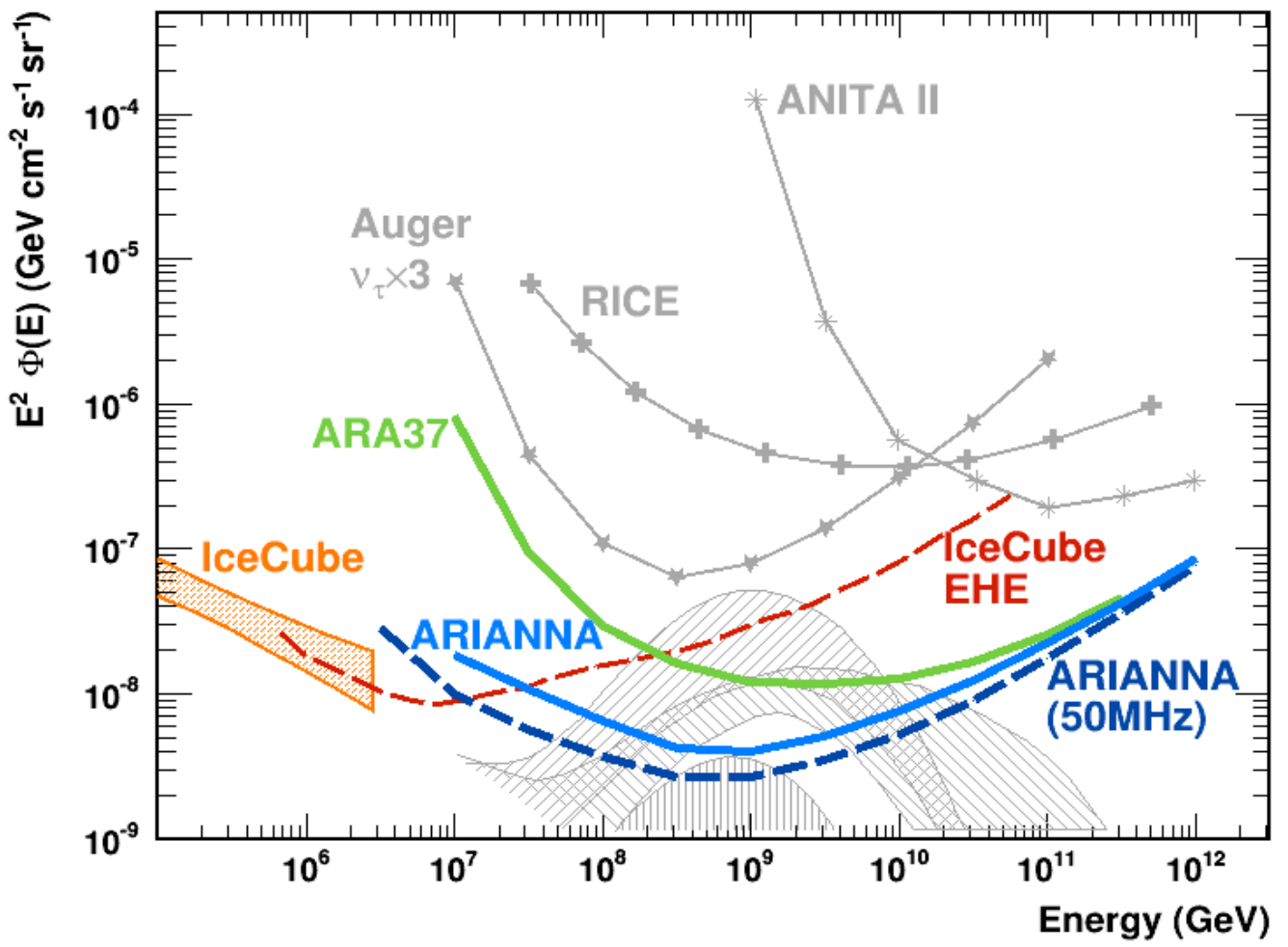

Figure 1: Representative survey of all-flavor neutrino differential flux limits (gray lines), assuming 1:1:1 flavor ratio where needed, and widely-discussed theoretical predictions for cosmogenic neutrinos (gray bands). Experimental limits from Auger [55], RICE [17], and ANITA [11] are shown in gray lines with symbols. ARIANNA (blue for $100 \mathrm{MHz}$ LPDA antennas and blue dash for $50 \mathrm{MHz}$ antennas) and ARA (green ARA37 curve) sensitivities are shown for 5 calendar years of operation. The IceCube signal (orange band) [37] and 5 year IceCube EHE limits (red dash) [30] are shown as well. See text for details the calculation of experimental sensitivities (figure adapted from [35]).

Both the ARIANNA and ARA37 sensitivity curves shown in Fig. 1 include operational livetime and analysis efficiency as currently reported by the pilot programs (rather than assuming idealistic perfection for both parameters, as usually plotted). In addition, to simplify comparison, this plot incorporates the same definitions for neutrino cross-section [53], energy binning, and assumed number of events in the absence of detection. The sensitivity of ARIANNA is factor 10 better than current 5yr limits of IceCube [29][30][36] at $10^{9} \mathrm{GeV}$, a useful benchmark for many cosmogenic models. In addition, ARIANNA will be able to measure neutrinos with potentially better angular resolution for the majority of detected events 
in IceCube at these energies. Given the current level of uncertainty in the models, the next generation of UHE neutrino telescopes must be flexible and powerful enough to observe potentially very small fluxes.

The IceCube Collaboration has reported a diffuse flux of neutrinos above $10 \mathrm{TeV}$ with a soft power law energy spectrum proportional to $\mathrm{E}^{-2.5}$ [37]. No cascade events are observed with energies above a few $\times 10^{6} \mathrm{GeV}$ in 5 years of observation. Interestingly, a track event was reported with a contained energy of a few $\mathrm{PeV}$, but due to possible energy loss outside of IceCube, the neutrino energy could be substantially larger than indicated by the contained energy [38]. Extending the contained event search to higher energies is difficult due to the finite geometry of IceCube and falling energy spectrum. IceCube researchers can switch strategies for events with much higher energies, but the effective volume above $10^{7} \mathrm{GeV}$ is less than optimal (see red dash curve in Fig. 1). There is strong interest by the community to improve the sensitivity for neutrino energies above $10^{7} \mathrm{GeV}$. As indicated Fig. 1, ARIANNA sensitivity smoothly joins the diffuse flux measured by IceCube.

If the best-fit power law spectrum of the IceCube signal extends to higher energies, ARIANNA would observe $\sim 4.8$ events in 5 calendar years of operation, with mean energy $\sim 10^{8}$ $\mathrm{GeV}$. On the other hand, if the energy spectrum of the IceCube events exhibits a cutoff, then neutrino events in ARIANNA indicate a new component, such as cosmogenic neutrinos or hard spectra source. It is all but certain that the discovery of a diffuse flux of neutrinos at extremely high energies would engender as much interest by the particle astrophysics community as currently enjoyed by the IceCube for its discovery of a diffuse flux of neutrinos.

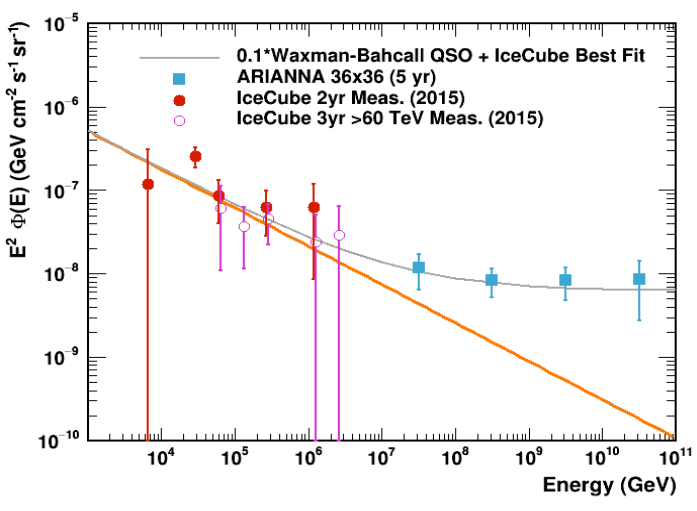

Figure 2:(left). Mock data assuming neutrino flux $\sim E^{-2}$ is normalized to $10 \%$ of WaxmanBahcall bound [39]. ARIANNA data are shown for 5 calendar years of operation (blue squares). The total number of events is 16.4. Current data from IceCube (closed and filled circles) and best fit energy spectrum (orange line) proportional to $E^{-2.5}$ also shown [37]. Note that IceCube data were reported per flavor, so the plotted points are multiplied by factor of 3 .

For more than a decade, theoretical modeling has highlighted the connection between the highest energy cosmic rays, presumably extragalactic in origin, and neutrinos at energies close to $10^{18} \mathrm{eV}$. Neutrino production by cosmic ray accelerators usually require surrounding material, such as gas or dust, that is neither too thin (or else too little production of neutrinos via $\mathrm{pp}$ or photohadronic interactions) nor too thick (otherwise, these sources cannot be responsible for the observed cosmic rays). In such conditions, accelerators should produce neutrino spectra that are nearly identical to cosmic ray spectra, which is thought to be proportional to $\mathrm{E}^{-2}$ to be consistent with the GZK cutoff and also first order Fermi acceleration models by relativistic shocks. The sweet-spot in surrounding density provides a constraint on the maximum diffuse flux of high energy neutrinos from cosmic ray sources, known as the Waxman-Bahcall bound. The benchmark value that we adopt for the total neutrino flux is 
$\mathrm{E}^{2 *} \mathrm{dN} / \mathrm{dE}<6.3 \times 10^{-8} \mathrm{GeV} / \mathrm{cm}^{2} / \mathrm{s} / \mathrm{sr}$. The next generation of extreme energy neutrino detectors can search for cosmic ray accelerators using neutrino surrogates, as evidenced by a hard spectrum, perhaps proportional $\sim \mathrm{E}^{-2}$ or slightly softer. At extreme energies, neutrino telescopes can measure relatively low flux normalizations due to the lack of physical backgrounds from atmospheric collisions. If indeed the IceCube neutrinos are not intimately connected to sources of extragalactic cosmic rays, then they constitute a physical background for this particular science goal. The energy threshold of radio-cherenkov neutrino detectors helps to suppress backgrounds from the sources of IceCube neutrinos. As illustrated in Fig. 2, the next generation of neutrino telescopes targeting extreme energies can observe a spectral hardening to $\mathrm{E}^{-2}$ for fluxes at $10 \%$ Waxman-Bahcall (assuming the absence of cosmogenic neutrinos in the same energy interval). Of course, it will be difficult to differentiate models with fluxes at the lower edge of sensitivity, but the positive identification of a nonzero flux at $\mathrm{E} \sim 10^{9} \mathrm{GeV}$ would set the sensitivity scale for future detectors. In addition, fluxes at this low a level would likely eliminate proton dominated cosmic ray fluxes from plausible cosmogenic neutrino models.

Although I have focused the discussion on hard-spectra and cosmogenic neutrino production, it is not the only potential science. For example, the cross-section and flavor ratio can be studied from features in the angular distributions [7][40]. In addition, ARIANNA and ARA can survey the southern half the sky for point sources of high-energy neutrinos from AGN or GRB with unprecedented sensitivity for energies between $10^{8}-10^{10} \mathrm{GeV}$. GNO provides similar coverage of the northern sky. Of course, the study of ultrahigh energy neutrinos with uniquely sensitive instruments could reveal completely unexpected phenomena.

\section{To summarize the unique science goals of radio-cherenkov neutrino telescopes:}

1. they can investigate the extreme energy component of IceCube neutrino spectrum.

2. they can probe the most pessimistic cosmogenic neutrino models for cosmic rays with mixed composition, as currently favored by Pierre Auger Observatory and Telescope Array, in addition to all proton-dominated models.

3. they can search for neutrino production by cosmic ray accelerators at fluxes approximately a factor 10 below the Waxman-Bahcall limit.

It should be emphasized that architecture of radio-based neutrino telescopes are modular and self-contained. Each station contributes to the integrated sensitivity, and partial arrays of stations provide significant capabilities. For example, the sensitivity of the initial construction phase of ARIANNA (with $10 \%$ of total number of stations) is comparable to IceCube at energies above $10^{17} \mathrm{eV}$, and both detectors view nearly the same sky. If a rare, perhaps once in a lifetime, explosive event occurs, then at least two detectors will observe the phenomena with complementary techniques and systematic errors. A transient event may provide the next significant advance in understanding, especially if optical and gravity wave telescopes observe the same event [41]. For such events, the angular precision of neutrino arrays will be superceded by the vastly superior pointing of optical instruments. For this reason, it is important to begin construction of radio-Cherenkov arrays as soon as possible. 


\section{Ongoing Development Efforts: The Contenders}

In this section we discuss several of the projects which target the detection of neutrinos with energies above $10^{16} \mathrm{eV}$. At these energies, propagation through the earth is strongly suppressed due to the increasing interaction cross-section, so neutrino telescopes can only observe neutrinos with nearly horizontal or downgoing trajectories. We begin with radio-based concepts that have successfully operated or currently operating a pilot program. At this conference, ANITA, ARIANNA and ARA collaborations presented new data from field operations. One of the major objectives of the ARIANNA and ARA37 pilot programs is to establish firm estimates for detector sensitivity under realistic conditions since this value relies on operating parameters such as analysis efficiency and livetime. Recent publications by both the ARA [15] and ARIANNA [14] collaborations and preliminary results from the completed Hexagonal Radio Array ( HRA, the pilot array of ARIANNA) [42] can be used to make a detailed comparison of the two techniques. ARA released results from two of three deep stations that ran in 2013 (one of the three deployed stations has not provided data since that time and so it was not included in the analysis) and the ARIANNA collaboration provided data from HRA, completed in December 2014, which corroborated results from an earlier investigation using data from the first three stations deployed in 2012. Since ARIANNA HRA stations transmit data in real time, it was possible to analyze the data acquired during the 2014/2015 austral summer.

\subsection{Antarctic Ross Iceshelf ANtenna Neutrino Array (ARIANNA)}

The idea of using a surface array of radio receivers to search for astrophysical sources has a long history [43]. The ARIANNA concept [14] utilizes the enormous Ross Ice Shelf near the coast of Antarctica to increase the sensitivity to ultrahigh energy cosmogenic neutrinos by roughly an order of magnitude when compared to the sensitivity of existing detectors such as IceCube (Fig. 1). ARIANNA exploits a fortuitous natural phenomenon: the water-ice interface at the bottom of the Ice Shelf reflects radio signals with remarkable fidelity [44]. Consequently, the reflected conical Cherekov pulses can be detected from neutrinos traveling in any downward direction that interact in the ice. The reflected (and occasionally direct) radio pulses are detected by autonomous antenna stations located within a few meters of top ice surface, which greatly simplifies deployment, maintenance and repair of a large array. All radio-based neutrino detectors in Antarctica must contend with shadowing effects from the surface gradient in the index of refraction. Fortunately, these effects in ARIANNA are mitigated by the mostly vertical paths taken by the reflected pulses. Moreover, ARIANNA capitalizes on several additional useful properties of the site: it is geographically close to McMurdo, the major US base in Antarctica, and it exhibits low levels of anthropogenic radio noise due to shielding from Minna Bluff and the Transantarctic Mountains. Due to the geographical proximity to McMurdo Station, the largest coastal base in Antarctica, cargo can be transported on land to the ARIANNA site by tracked vehicles, avoiding the need for costly flights by cargo aircraft and saving scarce resources.

ARIANNA consists of an array of stations, separated by $1 \mathrm{~km}$ on $36 \times 36$ grid. It views 0.5 Teratons of ice. In the baseline design, each station consists of eight single-polarization 
log-periodic antenna (LPDA) with measured gain and angular dependence [45]. Six LPDA point downward while two are canted upwards.

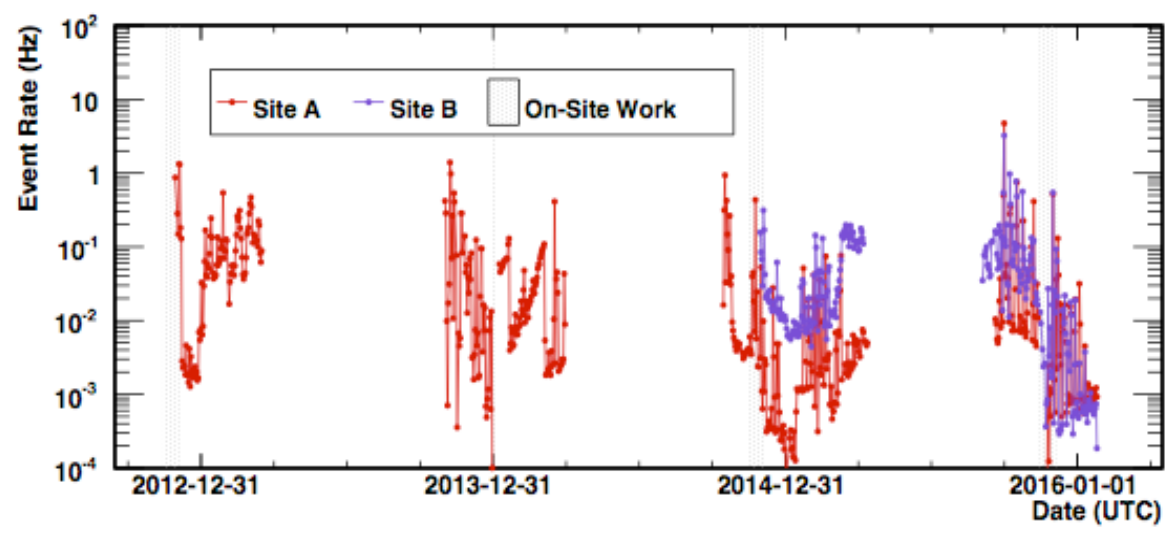

Figure 3: Trigger rates from two representative stations of ARIANNA HRA pilot program. The station at site $A$ was deployed in November 2012 and the station at site $B$ was deployed in November 2014.The average yearly live-time, including operational inefficiencies due to data transfer and calibration, is greater than 0.5 year. Since stations rely on solar panels for power, the livetime for HRA station is constrained to austral summers.

The downward LPDA are arranged to provide two orthogonal polarization measurements. The excellent directional sensitivity of LPDA insure that downgoing cosmic ray background events can be identified and discriminated from neutrino signals, which are upward traveling. If the cores of air showers strike the snow surface [46], additional discrimination is provided by the scattered signal from the firn layer. The US National Science Foundation approved a pilot program of 7 stations arranged in a hexagonal pattern on the snow surface, called the HRA. The HRA station has 4 LPDA, all pointed down, which is sufficient to reject background in the pilot array with high efficiency.

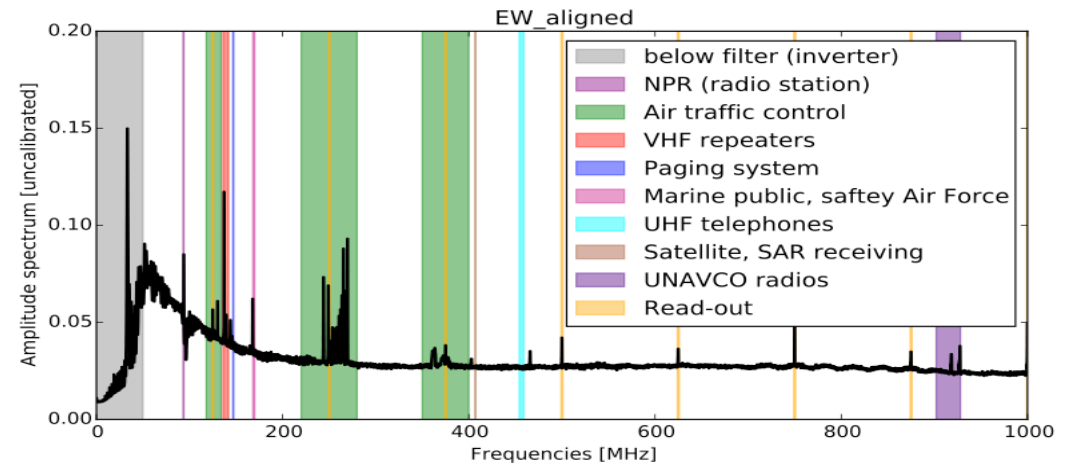

Figure 4: RFI survey at ARIANNA site between $50 \mathrm{MHz}$ and $1 \mathrm{GHz}$. The color band indicate known transmitters in the vicinity of McMurdo Station. The site survey also showed that the rate of impulsive noise was low, perhaps a more important characteristic of a suitable site.

Ice properties of ARIANNA site were measured [44] in studies involving short radio pulses reflected from the bottom of the ice and concluded that the site is suitable for ARIANNA. They showed: (1) the average attenuation length is $<\mathrm{L}>=470-140 \mathrm{f}$ meters (where $\mathrm{f}$ is frequency in GHz), (2) the field reflectivity of the ice-water surface at the bottom of the Ross ice Shelf is 
$0.7-1.0$ at the $68 \% \mathrm{CL}$, and (3) the fractional power scattering into the cross-polarized direction is $<5 \%$.

The first three stations of the ARIANNA HRA were deployed in November 2012 and completed in November 2014. Fig. 3 shows that the stations operated as expected and the recorded trigger rates are low, minimizing the amount of data that must be transmitted over the communication network. For the last few months shown in the figure, the onboard microprocessor performed low level event analysis, rejecting obvious narrowband noise from local transmitters. Consequently, the average trigger rates have decreased to $\sim 0.01 \mathrm{~Hz}$. HRA stations operated for more than $90 \%$ of the time the sun was above the horizon [54], consistent with design specifications.

An evaluation of the data collected from the stations during the past two years is quite encouraging [14][47]. The primary conclusions reached was that the site was RF quiet between $50 \mathrm{MHz}$ and $1 \mathrm{GHz}$ (see Fig. 4), justifying the design choice of $50 \mathrm{MHz}$ LPDA in future stations. Due to the radio quiet environment, non-thermal impulsive events were infrequent and readily identified. The efficiency of the analysis, defined as the ratio of neutrino events that survive analysis to the number of neutrino events at trigger level, was greater than $84 \%$ if averaged over energy. Non-thermal background events were largely confined to time periods involving high wind speeds, presumably correlated with storms. While some events had larger than thermal cross-correlation with the expected neutrino waveforms on a particular channel, none had characteristics of a neutrino signal in every antenna channel.

As seen in Fig. 1, the sensitivity of ARIANNA at energies $<10^{18} \mathrm{eV}$ diverges from ARA37, which is presumably due to several factors related to its unique surface location and two polarization design. ARIANNA employs large antennas with a high gain (6-8 dBi) over a very large bandwidth (between $50 \mathrm{MHz}$ and $1 \mathrm{GHz}$ ), and relatively narrow physical footprint of $5 \mathrm{~m}$ separation in the trigger requirements. This increases the geometric acceptance because the width of the emission pattern at a particular azimuthal angle on the Cherenkov cone can trigger an ARIANNA station. In contrast, ARA37 typically requires several points on the Cherenkov cone to intesect with a buried antennas in a given station. Also, for the lowest neutrino energies, the longer attenuation length of South Pole ice becomes less unimportant since most interaction vertices are nearby.

\subsection{Askaryan Radio Array (ARA37)}

The Askaryan Radio Array, ARA37, is sited at the South Pole within a few kilometers of IceCube [15]. The collaboration plans to deploy 37 stations, separated by $2 \mathrm{~km}$, to a depth of a depth of two hundred meters beneath the surface. Each station consists of 4 strings at the corners of a square grid with length $20 \mathrm{~m}$. The strings are instrumented with 4 bicone antennas at a depth between $180 \mathrm{~m}$ and $200 \mathrm{~m}$ to reduce the complication from ice density gradients near the surface on ray tracing. Despite the requirement to drill at least 148 holes, the logistical burden is less than required by the IceCube project because the holes are shallower and narrower. Currently, three stations have been deployed in the ice since the beginning of 2013, though one of the stations has only started to produce data in 2016. In an exposure time of 10 months, ARA reported a flux limit of $3 \times 10^{-6} \mathrm{GeV} \mathrm{cm}^{-2} \mathrm{~s}^{-1} \mathrm{sr}^{-1}$ at $10^{18} \mathrm{eV}$ from two stations. By comparison, in 4 months since commissioning HRA pilot array in December 2014, the ARIANNA collaboration reported a preliminary flux limit a factor of 4 larger [42]. Both results 
demonstrate the primary capabilities of the in-ice radio-cherenkov detectors to extract neutrino signals from thermal and anthropogenic backgrounds.

The ARA37 location provides advantages and disadvantages compared to ARIANNA. It exploits a much thicker ice sheet ( $3 \mathrm{~km}$ vs $0.5 \mathrm{~km}$ ) and longer attenuation lengths ( $>1 \mathrm{~km}$ vs $0.45 \mathrm{~km}$ at $100 \mathrm{MHz}$ ) due to colder ice, but ARIANNA compensates by instrumenting a larger area with more powerful antennas (higher gain, larger bandwidth) at an intrinsically quiet site. The surface location of the ARIANNA station relatively near McMurdo reduces construction and transportation burdens compared to ARA37, but on the other hand, electrical power can be reliably delivered all year to ARA37 from the South Pole Station. Until a robust wind-generator can be identified, ARIANNA operation is constrained to periods of the year when the sun rises above the horizon [51][52]. Now that both projects have realistic data from their respective pilot programs, it is possible to start the evaluation process of the relative merits of both approaches.

It was hoped that the close proximity between IceCube and ARA37 would lead to a measurable rate of co-observed events, but the high energy threshold of the radio telescopes, and the low rate of events at energies above $10^{16} \mathrm{eV}$ observed by IceCube has diminished that possibility. Also, it has been suggested that ARA37 can be triggered by IceCube to enhance its low energy capabilities, which is an important goal for all experimental efforts. The relative utility of this suggestion depends on the details of the geometrical overlap of the two detectors and the energy dependence of the effective apertures after the selection criteria are applied. An increase in the geometrical overlap could produce more RF interference from the IceCube PMTs and electronics in the surface counting house. It will be interesting to see the outcome of this calculation once the ARA collaboration finalizes its design.

\subsection{ANtarctic Impulse Transient Array (ANITA)}

ANITA, a long duration balloon mission first flown in 2006, is the most mature of the concepts discussed here [11]. A third flight of the ANITA payload was completed in January 2015 after 22 days, the shortest of the three missions [48]. From an altitude of $\sim 36 \mathrm{~km}$, ANITA detects neutrino-induced radio emission emerging from the ice sheet using a circular array of 48 dual polarized horn antennas. Radio pulses are expected to emerge from the ice with nearly vertical polarization. At balloon altitude, ANITA can survey an enormous circular patch of ice with a radius as large as $600 \mathrm{~km}$, but due to the complementary nature of the cherenkov cone geomery and total internal reflection by the firn ice-air boundary, the solid angle for neutrino detection is constrained to be within a few degrees of the local horizon of the ice surface. As seen in Fig. 1, the characteristic neutrino energy of ANITA is substantially larger than ARA37 and ARIANNA because the distance to the interaction vertex is typically $100 \mathrm{~km}$ rather than $1-2$ $\mathrm{km}$.

The first two missions of ANITA produced the best upper limits on the neutrino flux at energies above $10^{19} \mathrm{eV}$ [11]. In addition, ANITA observed pulsed radio emission from extensive air showers initiated by cosmic rays, a topic much discussed at this conference. In contrast to neutrino signals, the radio pulse from cosmic rays is expected to be horizontally polarized, due to the nearly vertical geomagnetic field in Antarctica. ANITA detected $\sim 20$ cosmic ray events with mean energy of $2.9 \times 10^{18} \mathrm{eV}$ [49]. A majority of these events reflected from the nonsmooth snow surface, and a few were detected directly through the atmosphere. To better understand the detailed scattering and reflectivity physics from the snow suface associated with 
cosmic ray events, the ANITA collaboration launched HiCal, a balloon-borne pulser that transmitted horizontally polarized RF impulses to the ANITA payload about $600 \mathrm{~km}$ distant. The analysis is ongoing.

As emphasized by the site location of ARIANNA, radio-cherenkov detectors require low RF noise environments to maximize trigger efficiency and simplify data analysis. This was the case for the first two ANITA flights, but over time, RF inteference from geostationary satellites have increased and the presence of unexpected noise reduced the efficiency of the exposure at levels not yet determined. The fourth ANITA flight is planned for December 2016. The payload will be upgraded with a programmable notch filter to help combat the enhanced levels of interference. With a bit of good fortune, it could achieve a factor 4 improvement in sensitivity relative to the third flight.

\subsubsection{New Ideas: Beamforming and Radio detection of Tau-neutrinos}

"Beam-forming" techniques to enhance signal to noise date back many years, and radio astronomers have used them with great success. However, implementation in radio-based neutrino telescopes has been hampered by requirements that phase coherence must be maintained to high levels for all viewing angles simultaneously though a dense and non-uniform medium such as polar ice. Also, beam-formed triggers will introduce significant bias in the angular sensitivity of the detector since it will magnify variations in angular response of the antenna. Undeterred, Vieregg, Bechtol, and Romero-Wolf [50] described a new idea to incorporate beam-form techniques in radio-cherenkov neutrino detectors. They and collaborators reported the first results of in situ testing at a Greenland site [16]. Researchers lowered a string of 8 dipole antennas to a depth of $115 \mathrm{~m}$, and sent a pulse from a transmitter at a distance of $168 \mathrm{~m}$ from the borehole. The time delay was manually adjusted to maximize signal to noise. Initial analysis focused on combining signals from two of the eight antennas, and results were consistent with expectation. It will be very interesting to see if signal to noise continues to improve with additional antennas and for a wide variety of incident angles.

Another recently developed detector concept, called the Giant Radio Array for Neutrino Detection (GRAND) [31], envisions an array of radio antennas spread over a much larger area than the current designs of polar neutrino detectors, though I should mention that the on-going development of polar detectors provides an avenue for growth to larger scales. In contrast to ARIANNA, which consists of 1296 stations covering an area of $10^{3} \mathrm{~km}^{2}$, GRAND consists of an array of $10^{5}$ radio antennas deployed over $200,000 \mathrm{~km}^{2}$ at a mountainous site. A significant challenge of the $R \& D$ phase of this project will be to prove adequate background rejection from cosmic rays and terrestrial sources at an estimated level of $10^{9}$ per year.

\section{Conclusions}

This past year, for the second time in 13 years, the Nobel committee returned the spotlight to neutrino physics, and the important role that non-accelerator based neutrino detectors played in those discoveries. It is crucial to continue to invest in the pursuit of the unknown with the potential for Nobel-worthy discoveries. Radio-cherenkov neutrino telescopes are designed to pursue the unknown in the neutrino sky at extreme energies, and the path forward is beginning to clarify. 
The ongoing operation of ARIANNA and ARA pilot arrays provides evidence that the most pressing technological and scientific challenges have been met. For example, commanding of the ARIANNA stations went smoothly, and the advanced trigger design provided 1-100 milli-Hz trigger rates with $4.0^{*} \mathrm{Vrms}$ thresholds. It was possible to reduce the data rates even further while maintaining sensitivity by installing on-station software tools to identify high priority events in real time. Stations operated routinely for 0.58 of a year on solar power including automatically restarting during sunrise, and hibernating during the dark winter. ARA stations receive power from the Amundsen-Scott station, so in principle, they can operate year round. Howerver, the reported livetime of the ARA pilot strings is only slightly larger than the ARIANNA pilot stations due to RF from air traffic during the summer and operational issues. Both groups expect improvement in this parameter.

Analysis of RF signals reflected from the bottom of the Ross Ice Shelf (ARIANNA) or sent from in-situ transmitters (ARA) demonstrate excellent angular reconstruction of the pulse direction. Cross-correlation techniques provide efficient rejection of thermal noise events, the vast majority of triggered events. Both ARA and ARIANNA have completed the search for high energy neutrinos with good efficiency. For example, the ARIANNA collaboration reported analysis efficiencies in excess of $84 \%$, nearly independent of energy. The efficiencies reported by ARA are somewhat lower, especially at lower energies. However, both collaboration expect these numbers to improve over time. Since the ARIANNA site is radio quiet and the geometric layout of the individual detector station is a relatively compact diameter of $5 \mathrm{~m}$, the procedure to reject background events is straightforward and efficient at all energies.

The next important milestone for ground based radio neutrino detectors will be the detection of cosmic rays, which can serve as an important component in the effort to validate the predictions of detector simulation tools, especially rejection rates of the background caused by the cosmic rays themselves. Cosmic ray initiated air showers constitute an ubiquitous background (and only) physic background, and analysis tools must be honed to identify these events and reliably differentiate them from neutrinos.

Given the encouraging results from the ARIANNA and ARA pilot programs, the time is right to begin the next stage of construction. Since ARA and ARIANNA consists of an array of independent stations, they can be constructed in a phased approach. The planned next step for ARIANNA expands the array to 100 stations - called ARIANNA-1. It will have sensitivity (averaged over the year) comparable to IceCube at $10^{18} \mathrm{eV}$, but with a superior pointing accuracy of 3 degrees for events at this energy, compared to $>10$ degrees for IceCube, facilitating the search for electromagnetic counterparts. Conversely, IceCube can measure the neutrino-induced cascades with much better energy resolution. Since both detectors view nearly the same region of sky at extreme energies, there is a strong possibility that both detectors will observe the same source. There is even greater power when the observations of both telescopes are combined. For example, the observation of a rare, explosive source in the Southern sky by two independent telescopes with different systematic uncertainties is almost a scientific necessity for such an important discovery with far-reaching consequences. ARIANNA-1 can be installed on a relatively short time scale to increase operational overlap with IceCube. In addition, it will lay the scientific and logistical groundwork for the 
construction of the full array of 1296 stations. The next phase of ARA construction includes the deployment of several additional stations, perhaps as early as 2017.

Looking further into the future, the ARIANNA collaboration plans to expland the array to 1296 stations. This array will achieve an order magnitude improvement in sensitivity compared to IceCube. ARIANNA will extend the search for the isotropic IceCube signal to higher energies and measure cosmogenic neutrinos and/or hard-spectra sources. The detection of any of these would have profound consequences. Similarly, ARA plans to expand the array to 37 stations with sensitivity shown in Fig. 1. One last point relevant to all radio-based polar detectors: in the search for diffuse emission, the flux is usually assumed to be invariant in time. Under these circumstances, it is not necessary to have all stations operational at the same time for 5 years. This mission critical science objective depends on the integrated observational time for all detector stations. The clock starts with the deployment of the first station.

\section{Acknowledgements}

This work was supported by the US National Science Foundation (both the Office of Polar Programs and the Physics Division) by grants ANT-08339133, NSF-0970175, and NSF1126672, the US Antarctic Program, and UC Irvine travel award.

\section{References}

[1] For general reviews, see G. Sigl, "Particle and Astrophysics Aspects of Ultrahigh Energy Cosmic Rays", Lect. Notes Phys. 556(2000)259 [astro-ph/0008364]; G. Sigl, "The Enigma of the Highest Energy Particles in Nature", Annals Phys. 303(2003)117 [astro-ph/0210049]; T. J. Weiler, "Extreme-Energy Cosmic Rays: Puzzles, Models, and Maybe Neutrinos", AIP Conf. Proc. 579 (2001) 58 [astro-ph/0103023]; E. Waxman, "Neutrino Astrophysics: A New Tool for Exploring the Universe", Science 315(2005)63.

[2] G. A. Askaryan JETP 14 (1962) 441; G. A. Askaryan JETP 21 (1965) 658.

[3] K. Greisen, Phys. Rev. Lett. 16(1966)748; G. T. Zatsepin and V.A. Kuzmin JETP Lett. 4(1966)78.

[4] F. W. Stecker (1973) Astrophys. Space Sci. 20 47; V. S. Berezinsky and A. Yu. Smirnov, (1975) Astroph. Space Sci. 32 461; V. S. Berezinsky and G. T. Zatsepin, Phys. Lett. 28B(1969)423.

[5] R. Engel, T. Stanev, and D. Seckel, "Neutrinos from Propagation of Ultra-high Energy Protons", Phys. Rev. D64(2001)093010; astro-ph/0101216; Seckel D and Stanev T (2005) Phys. Rev. Lett. 95141101.

[6] Barwick S W 2006 Using Neutrino Astronomy to Probe Physics at the Highest Energies Proc. Neutrino Oscillations in Venice (Venice, Italy, Edizioni Papergraf, ed. M. Baldo-Ceolin) 397-414.

[7] S. R. Klein and A. Connolly, "Neutrino Absorption in the Earth, Neutrino Cross-Sections, and New Physics”; [arXiv:1304.4891v1]

[8] S. T. Scully and F. Stecker, "Testing Lorentz Invariance with Neutrinos from Ultrahigh Energy Cosmic Ray Interactions,” Astropart. Phys. 34, 575 (2011).

[9] D. M. Mattingly, et al., "Possible Cosmogenic Neutrino Constraints on Planck-scale Lorentz Violation”, J. Cosmology Astropart. Phys. JCAP02 007 (2010); [arXiv:0911.0521]

[10] J. Ahrens J, et al. Phys. Rev. Lett. 92 (2004) 071102. 
[11] Gorham P W, et al. "Observational Constraints on the Ultra-high Energy Cosmic Neutrinos Flux from the Second Flight of the ANITA Experiment”, Phys. Rev. D82 (2010) 022004; Erratum: Gorham P W, et al., Phys. Rev. D85(2012)049901(E).

[12] M. Kestel (2004) Nucl Inst Meth A535 139; J. Ahrens et al. (2004) Astropart. Phys. 20 507; A. Achterberg et al. (2006) Astropart. Phys. 26155.

[13] I. Kravchenko et al. (2003) Astropart. Phys. 20 195; Kravchenko I, et al. (2006) Phys. Rev D73 082002 (astro-ph/0601148).

[14] S. W. Barwick, et al., (ARIANNA Collaboration), “A First Search for Cosmogenic Neutrinos with the ARIANNA Hexagonal Radio Array", Astropart. Phys., 70(2015)12; [arXiv:1410.7352]

[15] P. Allison, et al., (ARA Collaboration) "Performance of two Askaryan Radio Array stations and first results in the search for ultra-high energy neutrinos”, 2015, [arXiv:1507.0899v2]

[16] S. A. Wissel, et al., "Site Characterization and Detector Development for the Greenland Neutrino Observatory”, Proc. 34 ${ }^{\text {th }}$ Int. Cos. Ray Conf. ( Netherlands, 2015) PoS(ICRC2015) 828

[17] J. Abraham, et al., (Pierre Auger Collaboration), Phys. Rev. Lett. 101(2008)061101

[18] R. U. Abbasi, et al., (HiRes), Phys. Rev. Lett. 100(2008)101101, [astro-ph/0703099].

[19] Y. Tsunesada, et al., (Telescope Array Collaboration), Proc. $32^{\text {nd }}$ Int. Cosmic Ray Conf. (Beijing, China, 2011); [arXiv:1111.2507v1].

[20] T. Abu-Zayyad, et al., "The Cosmic Ray Energy Spectrum Observed with the Surface Detector of the Telescope Array Experiment”, Ap.J. 768 (2013)L1; [arXiv:1205.5067v3]

[21] D Allard, "Extragalactic Propagation fo Ultrahigh Energy Cosmic Rays”, Astropart. Phys. 39-40 (2012)33-43.

[22] P. L. Biermann and V. De Souza, "Centaurus A: The Extragalactic Source of Cosmic Rays Above the Knee”, Astrophys. J., 746(2012)72.

[23] D. Saltzberg, et al. 2001 Phys. Rev. Lett. 862802.

[24] P. W. Gorham, et al. 2006 “Observations of the Askaryan Effect in Ice” Phys. Rev. Lett. 99: (2007) 171101; (hep-ex/0611008).

[25] T. Huege, The Renaissance of Radio Detection of Cosmic Rays, Brazilian Journal of Physics, 44 (Oct. 2014) 520-529, [arxiv:1310.6927]

[26] M. Ackermann, et al., "Search for Ultra-High Energy Neutrinos with AMANDA-II”, Astrophys. J. 675(2008)1014.

[27] Pierre Auger Collaboration, "Limit on the diffuse flux of ultra-high energy tau neutrinos with the surface detector of the Pierre Auger Observatory”, Phys.Rev.D79(2009)102001.

[28] R.U. Abbassi, et al., [HiRes Collaboration], “An Upper Limit on the Electron-Neutrino Flux from the HiRes Detector”, Ap. J. 684 (2008) 790 [arXiv:0803.0554]; K. Martens, for the HiRes Collaboration,"HiRes Estimates And Limits for Neutrino Fluxes at the Highest Energies", Proc. Lepton Photon (2007) [arXiv:0707.4417]

[29] Karle A, "IceCube: Ultra high energy neutrinos" Proc. $25^{\text {th }}$ Int. Conf. Neutrino Phy. Astrophy. 2012 (Kyoto, Japan, 2012) Nucl. Phys. B 00(2012)1-7. 
[30] M. G. Aartsen, et al. (IceCube) “A Search for Extremely High Energy Neutrinos in 6 Years (20082014) of IceCube Data” PoS(ICRC2015)1064.; [arXiv:1510.05223]

[31] O. Martineau-Huynh, et al., The Giant Radio Array for Neutrino Detection, in proceedings of $34^{\text {th }}$ ICRC, (The Hague, Netherlands, 2015) [arXiv:1508.01919v1] .

[32] V. Berezinsky, Nucl. Phys. B Proc. Suppl. 151(2006)260.

[33] A. Aab, et al. (Pierre Auger collaboration), "The Pierre Auger Observatory: Contributions to the $34^{\text {th }}$ Int. Cos. Ray Conf. (Netherlands, 2015)”; [arXiv:1509.03732] See page 52.

[34] M. Fukushima (Telescope Array collaboration), "Recent results from Telescope Array", Proc. ISVHECRI (Cern, 2015); [arXiv:1503.06961]

[35] K.H. Kampert and M. Unger, "Measurements of the Cosmic Ray Composition with Air Shower Experiments”, Astropart. Phys. 35 (2012)660 ;[arXiv:1201.0018v2]

[36] M. G. Aartsen, et al. (IceCube Collaboration), "Probing the origin of cosmic rays with extremely high energy neutrinos using the IceCube Observatory”, Phys. Rev. D88 (2013)112008.

[37] M. G. Aartsen, et al., (IceCube collaboration) "A combined maximum-likelihood analysis of the high-energy astrophysical neutrino flux measured with IceCube”, Astrophys. J. 809(2015)98; [arXiv:1507.03991]

[38] S. Schoenen and L. Raedel (Icecube collaboration), "Detection of a multi-PeV neutrino-induced muon event from the Northern sky with IceCube”, The Astronomer's Telegram, ATel \#7856 (2015).

[39] E. Waxman and J. Bahcall, Astrophys. J. 541(2000)707 ; Waxman E and Bahcall J, "High Energy Neutrinos from Astrophysical Sources: An Upper Bound” Phys. Rev. D59(1999)023002

[40] S-H Wang, P.Chen, J. Nam, and M. Huang, "Feasibility of Determining Diffuse Ultra-High Energy Cosmic Neutrino Flavor Ratio through ARA Neutrino Observatory”, [arXiv:1302.1586]

[41] E. Waxman, “The Origin of IceCube's Neutrinos: Cosmic Ray Accelerators Embedded in Star Forming Calorimeters", Proc. Neutrino Astronomy (Eds. T. Gaisser and A. Karle, 2016); [arXiv:1511.00815]

[42] A. Halgren, for the ARIANNA Collaboration, “The ARIANNA Hexagonal Radio Array: Performance and Prospects" Proc. Very Large Volume Neutrino Telescopes (Rome, 2015).

[43] Gusev G A and Zheleznykh I M, "Neutrino and Muon Detection from the Radio Emission of Cascades Created by them in Natural Dielectric Media" JETP Lett. 38 (1983) 611.

[44] J. C. Hanson, S. W. Barwick, et al., "Radar Absorption, basal reflection, thickness, and polarization measurements from the Ross Ice Shelf, Antarctica”, J. Glaciol. 61(2015)438; [arXiv:1410.7134]

[45] S. W. Barwick, et al., "Time Domain Response of the ARIANNA Detector", Astropart. Phys, 62( 2015)139; [arXiv:1406.0820]

[46] K. D. de Vries, et al. “The Cosmic-Ray Air-Shower Signal in Askaryan Radio Detectors,” Astropart. Phys. 74 (2016) 96. ;[arXiv:1503.02808]

[47] S. W. Barwick (ARIANNA Collaboration), "Performance of the ARIANNA hexagonal radio array,” Proc. International Cosmic Ray Conference (Hague, Netherlands, 2015); [arXiv:1509.00109]

[48] S. Wissel (ANITA Collaboration), "Overview of the Third Flight of the ANITA Long-duration Balloon Payload", EoS (ICRC2015)1217 
[49] H. Schoorlemmer, et al., "Energy and Flux Measurements of Ultra-High Energy Cosmic Rays Observed During the First ANITA Flight”, Astropart. Phys. 77 (2016) 32; [arXiv:1506.05396v3]

[50] A. G. Vieregg, K. Bechtol, A. Romero-Wolf, “A new technique for detection of PeV neutrinos using a phased radio array”, submitted to JCAP, 2015; [arXiv:1504.08006v2]

[51] E. C. Berg, for the ARIANNA Collaboration, "Solar Wind and Power System for the ARIANNA Array”, Talk presented at Polar Technology Conf. (Annapolis, April, 2013)

[52] S.W. Barwick, et al., "Design and Performance of the ARIANNA HRA-3 Neutrino Detector Systems”,IEEE Trans.Nucl.Sci. 62(2015)2202; [arXiv:1410.7369]

[53] A. Connolly, R. S. Thorne and D. Waters, "Calculation of high energy neutrino-nucleon cross sections and uncertainties using the Martin-Stirling-Thorne-Watt parton distribution functions and implications for future experiments" Phys. Rev. D 83 (2011) 113009.

[54] S. W. Barwick, et al. (ARIANNA collaboration) "Livetime and sensitivity of the ARIANNA hexagonal radio array,” Proc. International Cosmic Ray Conference ( Netherlands, 2015); [arXiv:1509.00115]

[55] A. Aab, et al. (Pierre Auger collaboration), "An improved limit to the diffuse flux of ultra-high energy neutrinos from the Pierre Auger Observatory”, Phys. Rev. D91(2015)092008. 